\title{
Exact Solution of Asymmetric Diffusion With $N$ Classes of Particles of Arbitrary Size and Hierarchical Order
}

\author{
F. C. Alcaraz \\ Departamento de Física, Universidade Federal de São Carlos, 13565-905, São Carlos, SP Brazil \\ R. Z. Bariev \\ Departamento de Física, Universidade Federal de São Carlos, 13565-905, São Carlos, SP Brazil \\ The Kazan Physico-Technical Institute of the Russian Academy of Sciences, Kazan 420029, Russia
}

Received on 5 August, 2000

\begin{abstract}
The exact solution of the asymmetric exclusion problem with $N$ distinct classes of particles $(c=$ $1,2, \ldots, N)$, with hierarchical order is presented. In this model the particles (size 1) are located at lattice points, and diffuse with equal asymmetric rates, but particles in a class $c$ do not distinguish those in the classes $c^{\prime}>c$ from holes (empty sites). We generalize and solve exactly this model by considering the molecules in each distinct class $c=1,2, \ldots, N$ with sizes $s_{c}\left(s_{c}=0,1,2, \ldots\right)$, in units of the lattice spacing. The solution is derived via a Bethe ansatz of nested type.
\end{abstract}

\section{Introduction}

The similarity between the master equation describing time fluctuations in nonequilibrium problems and the Schrödinger equation describing the quantum fluctuations of quantum spin chains turns out to be fruitful for both areas of research [1]-[15]. Since many quantum chains are known to be exactly integrable through the Bethe ansatz, this provides exact information on the related stochastic model. At the same time classical physical intuition and probabilistic methods successfully applied to nonequilibrium systems give new insights into the physical and algebraic properties of quantum chains.

An example of this fruitful interchange is the problem of asymmetric diffusion of hard-core particles on the one dimensional lattice ( see $[16,17,18]$ for reviews). This model is related to the exactly integrable anisotropic Heisenberg chain in its ferromagnetic regime [19] (XXZ model). However if we demand this quantum chain to be invariant under a quantum group symmetry $U_{q}(S U(2))$, we have to introduce, for the equilibrium statistical system, unusual surface terms, which on the other hand have a nice and simple interpretation for the related stochastic system [3, 4].

In the area of exactly integrable models it is well known that one of the possible extensions of the spin- $\frac{1}{2} \mathrm{XXZ}$ chain to higher spins is the anisotropic spin-S Sutherland model (grading $\epsilon_{1}=\epsilon_{2}=\ldots=\epsilon_{2 s+1}=1$ ) [20]. On the other hand in the area of diffusion limited reactions a simple extension of the asymmetric diffusion problem is the problem of diffusion with particles belonging to $N$ distinct classes $(c=1,2, \ldots, N)$ with hierarchical order [22]-[24] . In this problem a mixture of hard-core particles diffuses on the lattice. Particles belonging to a class $c(c=1, \ldots, N)$ ignore the presence of those in classes $c^{\prime}>c$, i.e., they see them in the same way as they see the holes (empty sites). In [3] it was shown that for open boundary conditions the anisotropic spin-1 Sutherland model and this last stochastic model, in the case $N=2$, are exactly related. The Hamiltonian governing the quantum or time fluctuations of both models is given in terms of generators of a Hecke algebra, invariant under the quantum group $U_{q} S U(3)$. In fact this relation can be extended to arbitrary values of $N$, and the quantum chain associated to the stochastic model is invariant under the quantum $U_{q}(S U(N+1))$ group. In this paper we derive through the Bethe ansatz the exact solution of the associated quantum chain, on a closed lattice. Recently [15] (see also [14]) we have shown that without losing its exact integrability, we can consider the problem of asymmetric diffusion with an arbitrary mixture of molecules with different sizes (even zero), as long they do not in- 
terchange positions, that is, there is no reactions. In this paper we extend the asymmetric diffusion problem with $N$ types of particles with hierarchical order, to the case where the particles in each class have an arbitrary size, in units of the lattice spacing. Unlike the case of asymmetric diffusion problem, we have in this case a nested Bethe ansatz [25]. A pedagogical presentation for the simplest case $N=2$ was presented in [26].

The paper is organized as follows. In the next section we introduce the generalized asymmetric model with $N$ types of particles with hierarchical order and derive the associated quantum chain. In section 3 the Bethe ansatz solution of the model is presented. Finally in section 4 we present our conclusions, with some possible generalizations of the stochastic problem considered in this paper, and some perspectives on future work.

\section{The generalized asymmetric diffusion model with $N$ classes of particles with hierarchical order}

A simple extension of the asymmetric exclusion model, in which hard-core particles diffuse on the lattice, is the problem where a mixture of particles belonging to different classes $(c=1,2, \ldots, N)$ diffuses on the lattice.. This problem in the case where we have only $N=2$ classes was used to describe shocks [22]-[24] in nonequilibrium and also has a stationary probability distribution that can be expressed via the matrixproduct ansatz [27]. In [28] it was also shown that the stationary state of the case $N=3$ can also be expressed by the matrix-product ansatz. In this model we have $n_{1}, n_{2}, \ldots n_{N}$ molecules belonging to the classes $c=1,2, \ldots, N$, respectively. All classes of molecules diffuse asymmetrically, but with the same asymmetrical rates, whenever they encounter empty sites (holes) at nearest-neighbor sites. However, when molecules of different classes, $c$ and $c^{\prime}\left(c<c^{\prime}\right)$, are at their minimum separation, the molecules of class $c$ exchange position with the same rate as they diffuse, and consequently the molecules in the class $c$ see no difference between molecules belonging to the classes $c^{\prime}>c$ and holes.
We now introduce a generalization of the above model, where instead of having unit size, the molecules in each distinct class $c=1,2, \ldots, N$ have in general distinct sizes $s_{1}, s_{2}, \ldots, s_{N}\left(s_{1}, \ldots, s_{N}=1,2, \ldots\right)$, respectively, in units of lattice spacing. In Fig. 1 we show some examples of molecules of different sizes. We may think of a molecule of size $s$ as formed by $s$ monomers (size 1), and for simplicity, we define the position of the molecule as the center of its leftmost monomer. The molecules have a hard-core repulsion: the minimum distance $d_{\alpha \beta}$, in units of the lattice spacing, between molecules $\alpha$ and $\beta$, with $\alpha$ in the left, is given by $d_{\alpha \beta}=s_{\alpha}$. In order to describe the occupancy of a given configuration of molecules we define at each lattice site $i(i=1,2, \ldots, L)$ a variable $\beta_{i}(i=1,2, \ldots, L)$, taking the values $\beta_{i}=0,1, \ldots, N$. The values $\beta=1,2, \ldots, N$ represent sites occupied by molecules of class $c=1,2, \ldots, N$, respectively. On the other hand the value $\beta=0$ represents an empty site or an excluded one, due to the finite size of the molecules. As an example, in a chain of $L=8$ sites, the configuration in which a particle of class 1 , with size $s_{1}=2$ is at site 1 , and another particle, of class 2 , with size $s_{2}=3$ is at site 3 , is represented by $\{\beta\}=\{1,0,0,2,0,0,0,0\}$. Thus the allowed configurations are given by the set $\left\{\beta_{i}\right\}(i=1, \ldots, L)$, where for each pair $\left(\beta_{i}, \beta_{j}\right) \neq 0$ with $j>i$ we should have $j-i \geq s_{\beta_{i}}$.

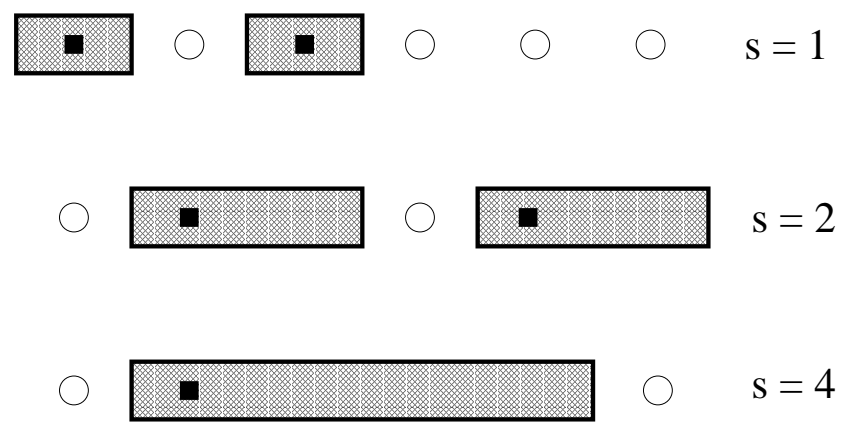

Figura.1 Example of configurations of molecules with distinct sizes $s$ in a lattice of size $L=6$. The coordinates of the molecules are denoted by the black squares.

The time evolution of the probability distribution $P(\{\beta\}, t)$, of a given configuration $\{\beta\}$ is given by the master equation

$$
\frac{\partial P(\{\beta\}, t)}{\partial t}=\sum_{\left\{\beta^{\prime}\right\}}\left[-\Gamma\left(\{\beta\} \rightarrow\left\{\beta^{\prime}\right\}\right) P(\{\beta\}, t)+\Gamma\left(\left\{\beta^{\prime}\right\} \rightarrow\{\beta\}\right) P\left(\left\{\beta^{\prime}\right\}, t\right),\right]
$$


where $\Gamma\left(\{\beta\} \rightarrow\left\{\beta^{\prime}\right\}\right)$ is the transition rate for configuration $\{\beta\}$ to change to $\left\{\beta^{\prime}\right\}$. In the present model we only allow, whenever the constraint of excluded volume is satisfied, the particles to diffuse to nearest-neighbor sites, or to exchange positions. The possible motions are diffusion to the right

$$
\beta_{i} \emptyset_{i+1} \rightarrow \emptyset_{i} \beta_{i+1}, \quad(\beta=1, \ldots, N) \quad\left(\text { rate } \Gamma_{R}\right)
$$

diffusion to the left

$\emptyset_{i} \beta_{i+1} \rightarrow \beta_{i} \emptyset_{i+1}, \quad(\beta=1, \ldots, N)$ $\left(\right.$ rate $\left.\Gamma_{L}\right)$ same rate as they interchange positions with the empty sites (diffusion). We should remark however that unless the particles in class $c^{\prime}$ have unit size $\left(s_{c}=1\right)$, the net effect of these particles in those of class $c$ is distinct from the effect produced by the holes, since as the result of the exchange the particles in class $c$ will move by $s_{c^{\prime}}$ lattice size units, accelerating its diffusion.

The master equation (1) can be written as a Schrödinger equation in Euclidean time (see Ref. [3] for general application for two body processes)

and interchange of particles

$$
\frac{\partial \mid P>}{\partial t}=-H \mid P>
$$

$\beta_{i} \beta_{i+s_{\beta}}^{\prime} \rightarrow \beta_{i}^{\prime} \beta_{i+s_{\beta^{\prime}}}, \quad\left(\beta<\beta^{\prime}=1, \ldots, N\right) \quad\left(\right.$ rate $\left.\Gamma_{R}\right)$ if we interpret $\mid P>\equiv P(\{\beta\}, t)$ as the associated $\beta_{i} \beta_{i+s_{\beta}}^{\prime} \rightarrow \beta_{i}^{\prime} \beta_{i+s_{\beta^{\prime}}}, \quad\left(\beta>\beta^{\prime}=1, \ldots, N\right) \quad($ rate $\Gamma(4)$ )

As we see from (4), particles belonging to a given class $c$ interchange positions with those of class $c^{\prime}>c$ with the

(4)) wave function. If we represent $\beta_{i}$ as $\mid \beta>_{i}$ the vector $\left|\beta>_{1} \otimes\right| \beta>_{2} \otimes \cdots \otimes \mid \beta>_{L}$ will give us the associated Hilbert space. The process (2)-(4) gives us the Hamiltonian (see Ref. [3] for general applications)

$$
\begin{aligned}
H= & D \sum_{j} H_{j} \\
H_{j}= & -\mathcal{P}\left\{\sum_{\alpha=1}^{N}\left[\epsilon_{+}\left(E_{j}^{0 \alpha} E_{j+1}^{\alpha 0}-E_{j}^{\alpha \alpha} E_{j+1}^{00}\right)+\epsilon_{-}\left(E_{j}^{\alpha 0} E_{j+1}^{0 \alpha}-E_{j}^{00} E_{j+1}^{\alpha \alpha}\right)\right]\right. \\
& \left.+\sum_{\alpha=1}^{N} \sum_{\beta=1}^{N} \epsilon_{\alpha, \beta}\left(E_{j}^{\beta \alpha} E_{j+s_{\beta}}^{\alpha 0} E_{j+s_{\alpha}}^{0 \beta}-E_{j}^{\alpha \alpha} E_{j+s_{\beta}}^{00} E_{j+s_{\alpha}}^{\beta \beta}\right)\right\} \mathcal{P}
\end{aligned}
$$

with

$$
D=\Gamma_{R}+\Gamma_{L}, \quad \epsilon_{+}=\frac{\Gamma_{R}}{\Gamma_{R}+\Gamma_{L}}, \quad \epsilon_{-}=\frac{\Gamma_{L}}{\Gamma_{R}+\Gamma_{L}} \quad\left(\epsilon_{+}+\epsilon_{-}=1\right)
$$

$$
\epsilon_{\alpha \beta}= \begin{cases}\epsilon_{+} & \alpha<\beta \\ 0 & \alpha=\beta \\ \epsilon_{-} & \alpha>\beta\end{cases}
$$

and periodic boundary conditions. The matrices $E^{\alpha, \beta}$ are $(N+1) \times(N+1)$ matrices with a single nonzero element $\left(E^{\alpha, \beta}\right)_{i, j}=\delta_{\alpha, i} \delta_{\beta, j}(\alpha, \beta, i, j=0, \ldots, N)$. The projector $\mathcal{P}$ in (6), projects out from the associated Hilbert space the vectors $\mid\{\beta\}>$ which represent forbidden positions of the molecules due to their finite size, which mathematically means that for all $i, j$ with $\beta_{i}, \beta_{j} \neq 0, \quad|i-j| \geq s_{\beta_{i}} \quad(j>i)$. The constant $D$ in (6) fixes the time scale; for simplicity we chose $D=1$. A particular simplification of (6) occurs when the molecules in all classes have the same size $s_{1}=s_{2}=\ldots=s_{N}=s$. In this case the Hamiltonian can be expressed as an anisotropic nearest-neighbor interaction spin- $N / 2 S U(N+1)$ chain. Moreover in the case where their sizes are unity $(s=1)$ the model can be related to the anisotropic version [21] of the $S U(N+1)$ Sutherland model [20] with twisted boundary conditions.

\section{The Bethe ansatz equations}

We present in this section the exact solution of the general quantum chain (6). A pedagogical presentation for the particular case where $N=2$ was presented in [26].

Due to the conservation of particles in the diffusion and interchange processes the total number of particles $n_{1}, n_{2}, \ldots, n_{N}$ in each class are good quantum numbers and consequently we can split the associated Hilbert space into block disjoint sectors labeled by the numbers $n_{1}, n_{2} \ldots n_{N}\left(n_{i}=0,1, \ldots ; i=1, \ldots, N\right)$. We therefore 
consider the eigenvalue equation

$$
H\left|n_{1}, n_{2}, \ldots, n_{N}>=E\right| n_{1}, n_{2} \ldots, n_{N}>,
$$

$$
\left|n_{1}, n_{2}, \ldots, n_{N}>=\sum_{\{Q\}} \sum_{\{x\}} f\left(x_{1}, Q_{1} ; \ldots ; x_{n}, Q_{n}\right)\right| x_{1}, Q_{1} ; \ldots ; x_{n}, Q_{n}>
$$

and $n=\sum_{i=1}^{N} n_{i}$ is the total number of particles. In (10) $\mid x_{1}, Q_{1} ; \ldots ; x_{n}, Q_{n}>$ means the configuration where a particle of class $Q_{i}\left(Q_{i}=1,2, \ldots, N\right)$ is at position $x_{i}\left(x_{i}=1, \ldots, L\right)$. The summation $\{Q\}=\left\{Q_{1}, \ldots, Q_{n}\right\}$ extends over all permutations of the $n$ integer numbers $\{1,2, \ldots, N\}$ in which $n_{i}$ terms have the value $i(i=1,2, \ldots, N)$, while the summation $\{x\}=\left\{x_{1}, \ldots, x_{n}\right\}$ runs, for each permutation $\{Q\}$, in the set of the $n$ nondecreasing integers satisfying

$$
\begin{gathered}
x_{i+1} \geq x_{i}+s_{Q_{i}}, \quad i=1, \ldots, n-1, \\
s_{Q_{1}} \leq x_{n}-x_{1} \leq N-s_{Q_{n}} .
\end{gathered}
$$

Before getting the results for general values of $n$ let us consider initially the cases where we have 1 or 2 particles.

$\mathbf{n}=\mathbf{1}$. For one particle on the chain, in any class $c=1,2, \ldots, N$, as a consequence of the translational invariance of (6) it is simple to verify directly that the eigenfunctions are the momentum- $k$ eigenfunctions

$$
\left|0, \ldots, 0,1_{c}, 0, \ldots, 0>=\sum_{x=1}^{L} f(x, c)\right| x, c>, \quad c=1, \ldots, N
$$

with

$$
f(x, c)=e^{i k x}, \quad k=\frac{2 \pi l}{L}, \quad l=0,1, \ldots, L-1,
$$

and energy given by

$$
E=e(k) \equiv-\left(\epsilon_{-} e^{i k}+\epsilon_{+} e^{-i k}-1\right) .
$$

$\mathbf{n}=\mathbf{2}$. For two particles of classes $Q_{1}$ and $Q_{2}$ $\left(Q_{1}, Q_{2}=1,2, \ldots, N\right)$ on the lattice, the eigenvalue equation (9) gives us two distinct relations depending on the relative location of the particles. The first relation applies to the case in which a particle of class $Q_{1}$ $\left(\right.$ size $\left.s_{Q_{1}}\right)$ is at position $x_{1}$ and a particle $Q_{2}\left(\operatorname{size} s_{Q_{2}}\right)$ is at position $x_{2}$, where $x_{2}>x_{1}+s_{Q_{1}}$. We obtain in this case the relation

$$
\begin{aligned}
E f\left(x_{1}, Q_{1} ; x_{2}, Q_{2}\right) & =-\epsilon_{+} f\left(x_{1}-1, Q_{1} ; x_{2}, Q_{2}\right)-\epsilon_{-} f\left(x_{1}, Q_{1} ; x_{2}+1, Q_{2}\right) \\
-\epsilon_{-} f\left(x_{1}+1, Q_{1} ; x_{2}, Q_{2}\right) & -\epsilon_{+} f\left(x_{1}, Q_{1} ; x_{2}-1, Q_{2}\right)+2 f\left(x_{1}, Q_{1} ; x_{2}, Q_{2}\right),
\end{aligned}
$$

where we have used the relation $\epsilon_{+}+\epsilon_{-}=1$. This last equation can be solved promptly by the ansatz

$$
\begin{aligned}
f\left(x_{1}, Q_{1} ; x_{2}, Q_{2}\right) & =\sum_{P} A_{P_{1}, P_{2}}^{Q_{1}, Q_{2}} e^{i\left(k_{P_{1}} x_{1}+k_{P_{2}} x_{2}\right)} \\
& =A_{1,2}^{Q_{1}, Q_{2}} e^{i\left(k_{1} x_{1}+k_{2} x_{2}\right)}+A_{2,1}^{Q_{1}, Q_{2}} e^{i\left(k_{2} x_{1}+k_{1} x_{2}\right)}
\end{aligned}
$$

with energy

$$
E=e\left(k_{1}\right)+e\left(k_{2}\right),
$$

where $k_{1}, k_{2}, A_{1,2}^{Q_{1}, Q_{2}}$ and $A_{2,1}^{Q_{1}, Q_{2}}$ are free parameters to be fixed. In (16) the summation is over the permutations $P=P_{1}, P_{2}$ of $(1,2)$. The second relation applies when $x_{2}=x_{1}+s_{Q_{1}}$. In this case instead of (15) we have

$$
\begin{array}{ll}
E f\left(x_{1}, Q_{1} ; \quad\right. & \left.x_{1}+s_{Q_{1}}, Q_{2}\right)=-\epsilon_{+} f\left(x_{1}-1, Q_{1} ; x_{1}+s_{Q_{2}}, Q_{2}\right)-\epsilon_{-} f\left(x_{1}, Q_{1} ; x_{1}+s_{Q_{1}}+1, Q_{2}\right) \\
& -\tilde{\epsilon}_{Q_{2}, Q_{1}} f\left(x_{1}, Q_{2} ; x_{1}+s_{Q_{2}}, Q_{1}\right)+\left(1+\tilde{\epsilon}_{Q_{1}, Q_{2}}\right) f\left(x_{1}, Q_{1} ; x_{1}+s_{Q_{1}}, Q_{2}\right) .
\end{array}
$$

If we now substitute the ansatz (16) with the energy (17), the constants $A_{12}^{Q_{1}, Q_{2}}$ and $A_{21}^{Q_{1}, Q_{2}}$, initially arbitrary, should now satisfy 


$$
\sum_{P}\left\{\left[\mathcal{D}_{P_{1}, P_{2}}+e^{i k_{P_{2}}}\left(1-\tilde{\epsilon}_{Q_{1}, Q_{2}}\right)\right] e^{i k_{P_{2}}\left(s_{Q_{1}}-1\right)} A_{P_{1}, P_{2}}^{Q_{1}, Q_{2}}+\tilde{\epsilon}_{Q_{2}, Q_{1}} e^{i k_{P_{2}} s_{Q_{2}}} A_{P_{1}, P_{2}}^{Q_{2}, Q_{1}}\right\}=0
$$

where

$$
\mathcal{D}_{l, m}=-\left(\epsilon_{+}+\epsilon_{-} e^{i\left(k_{l}+k_{m}\right)}\right) .
$$

At this point it is convenient to consider separately the case where $Q_{1}=Q_{2}$ from those where $Q_{1} \neq Q_{2}$. If $Q_{1}=Q_{2}=Q(Q=1, \ldots, N)$ eq. (19) gives

$$
\sum_{P}\left(\mathcal{D}_{P_{1}, P_{2}}+e^{i k_{P_{2}}}\right) e^{i k_{P_{2}}\left(s_{Q}-1\right)} A_{P_{1}, P_{2}}^{Q, Q}=0
$$

and the cases $Q_{1} \neq Q_{2}$ give us the equations

$$
\sum_{P}\left[\begin{array}{cc}
\mathcal{D}_{P_{1}, P_{2}}+e^{i k_{P_{2}} \epsilon_{Q_{2}, Q_{1}}} & \epsilon_{Q_{2}, Q_{1}} e^{i k_{P_{2}}} \\
\epsilon_{Q_{1}, Q_{2}} e^{i k_{P_{2}}} & \mathcal{D}_{P_{1}, P_{2}}+\epsilon_{Q_{1}, Q_{2}} e^{i k_{P_{2}}}
\end{array}\right]\left[\begin{array}{c}
e^{i k_{P_{2}}\left(s_{Q_{1}}-1\right)} A_{P_{1}, P_{2}}^{Q_{1}, Q_{2}} \\
e^{i k_{P_{2}}\left(s_{Q_{2}}-1\right)} A_{P_{1}, P_{2}}^{Q_{2}, Q_{1}}
\end{array}\right]=0 .
$$

Performing the above summation we obtain, after lengthy but straightforward algebra, the following relation among the amplitudes

$$
\begin{aligned}
{\left[\begin{array}{c}
A_{1,2}^{Q_{1}, Q_{2}} e^{i k_{2}\left(s_{Q_{1}}-1\right)} \\
A_{1,2}^{Q_{2}, Q_{1}} e^{i k_{2}\left(s_{Q_{2}}-1\right)}
\end{array}\right] } & =-\frac{\mathcal{D}_{1,2}+e^{i k_{1}}}{\mathcal{D}_{1,2}+e^{i k_{2}}} \\
& \times\left\{1-\Phi\left(k_{1}, k_{2}\right)\left[\begin{array}{cc}
\epsilon_{Q_{1}, Q_{2}} & -\epsilon_{Q_{2}, Q_{1}} \\
-\epsilon_{Q_{1}, Q_{2}} & \epsilon_{Q_{2}, Q_{1}}
\end{array}\right]\right\}\left[\begin{array}{c}
A_{2,1}^{Q_{1}, Q_{2}} e^{i k_{1}\left(s_{Q_{1}}-1\right)} \\
A_{2,1}^{Q_{2}, Q_{1}} e^{i k_{1}\left(s_{Q_{2}}-1\right)}
\end{array}\right]
\end{aligned}
$$

where

$$
\Phi\left(k_{1}, k_{2}\right)=\frac{e^{i k_{1}}-e^{i k_{2}}}{\mathcal{D}_{1,2}+e^{i k_{1}}} .
$$

Equations (21) and (22) can be written in a compact form

$$
A_{P_{1}, P_{2}}^{Q 1, Q 2}=-\Xi_{P_{1}, P_{2}} \sum_{Q_{1}^{\prime}, Q_{2}^{\prime}=1}^{N} S_{Q_{1}^{\prime}, Q_{2}^{\prime}}^{Q_{1}, Q_{2}}\left(k_{P_{1}}, k_{P_{2}}\right) A_{P_{2}, P_{1}}^{Q_{2}^{\prime}, Q_{1}^{\prime}}, \quad\left(Q_{1}, Q_{2}=1, \ldots, N\right)
$$

with

$$
\Xi_{l, j}=\frac{\mathcal{D}_{l, j}+e^{i k_{l}}}{\mathcal{D}_{l, j}+e^{i k_{j}}}=\frac{\epsilon_{+}+\epsilon_{-} e^{i\left(k_{l}+k_{j}\right)}-e^{i k_{l}}}{\epsilon_{+}+\epsilon_{-} e^{i\left(k_{l}+k_{j}\right)}-e^{i k_{j}}},
$$

where we have introduced the $S$ matrix. From (21) and (22) this $\mathrm{S}$ matrix has only $N(2 N-1)$ non zero elements, namely

$$
\begin{aligned}
& S_{Q_{2}, Q_{1}}^{Q_{1}, Q_{2}}\left(k_{1}, k_{2}\right)=\left[1-\epsilon_{Q_{1}, Q_{2}} \Phi\left(k_{1}, k_{2}\right)\right] e^{i\left(k_{1}-k_{2}\right)\left(s_{Q_{1}}-1\right)} \quad\left(Q_{1}, Q_{2}=1, \ldots, N\right) \\
& S_{Q_{1}, Q_{2}}^{Q_{1}, Q_{2}}\left(k_{1}, k_{2}\right)=\epsilon_{Q_{2}, Q_{1}} \Phi\left(k_{1}, k_{2}\right) e^{i k_{1}\left(s_{Q_{2}}-1\right)} e^{-i k_{2}\left(s_{Q_{1}}-1\right)} \quad\left(Q_{1}, Q_{2}=1, \ldots, N ; Q_{1} \neq Q_{2}\right) .
\end{aligned}
$$

Equations (23) do not fix the "wave numbers" $k_{1}$ and $k_{2}$. In general, these numbers are complex, and are fixed due to the cyclic boundary condition

$$
f\left(x_{1}, Q_{1} ; x_{2}, Q_{2}\right)=f\left(x_{2}, Q_{2} ; x_{1}+N, Q_{1}\right)
$$

which from (16) give the relations

$$
A_{1,2}^{Q_{1} Q_{2}}=e^{i k_{1} N} A_{2,1}^{Q_{2}, Q_{1}}, \quad A_{2,1}^{Q_{1}, Q_{2}}=e^{i k_{2} N} A_{2,1}^{Q_{2}, Q_{1}}
$$

This last equation, when solved by exploiting (23)-(25), gives us the possible values of $k_{1}$ and $k_{2}$, and from (17) the eigenenergies in the sector with 2 particles. Instead of solving these equations for the particular case $n=2$ let us now consider the case of general $n$. 
General $\mathbf{n}$. The above calculation can be generalized for arbitrary occupation $\left\{n_{1}, n_{2}, \ldots, n_{N}\right\}$ of particles in classes $1,2, \ldots, N$, respectively. The ansatz for the wave function (10) becomes

$$
f\left(x_{1}, Q_{1} ; \ldots ; x_{n}, Q_{n}\right)=\sum_{P} A_{P_{1}, \ldots, P_{n}}^{Q_{1}, \ldots, Q_{n}} e^{i\left(k_{P_{1}} x_{1}+\cdots+k_{P_{n}} x_{n}\right)},
$$

where the sum extends over all permutations $P$ of the integers $1,2, \ldots, n$, and $n=\sum_{i=1}^{N} n_{i}$ is the total number of particles.

Application of the translation operator to the above wave functions implies that (10) are also eigenfunctions of the momentum operator with eigenvalues

$$
p=\sum_{j=1}^{n} k_{j}=\frac{2 \pi l}{L}, \quad(l=0,1, \ldots, L-1) .
$$

For the components $\mid x_{1}, Q_{1} ; \ldots ; x_{n}, Q_{n}>$ where $x_{i+1}-x_{i}>s_{Q_{i}}$ for $i=1,2, \ldots, n$, it is simple to see that the eigenvalue equation (9) is satisfied by the ansatz (28) with energy

$$
E=\sum_{j=1}^{n} e\left(k_{j}\right)
$$

On the other hand if a pair of particles of class $Q_{i}, Q_{i+1}$ is at positions $x_{i}, x_{i+1}$, where $x_{i+1}=x_{i}+s_{Q_{i}}$, equation (9) with the ansatz (28) and the relation (30) give us the generalization of relation (23), namely

$$
A_{\ldots, P_{i}, P_{i+1}, \ldots}^{\cdots, Q_{i}, Q_{i+1}, \cdots}=-\Xi_{P_{i}, P_{i+1}} \sum_{Q_{1}^{\prime}, Q_{2}^{\prime}}^{N} S_{Q_{1}^{\prime}, Q_{2}^{\prime}}^{Q_{i}, Q_{i+1}}\left(k_{P_{i}}, k_{P_{i+1}}\right) A_{\ldots, P_{i+1}, P_{i}, \ldots}^{\cdots, Q_{2}^{\prime}, Q_{1}^{\prime}, \ldots} \quad\left(Q_{i}, Q_{i+1}=1,2, \ldots, N\right)
$$

with $S$ given by eq. (25). Inserting the ansatz (28) in the boundary condition

$$
f\left(x_{1}, Q_{1} ; \ldots ; x_{n}, Q_{n}\right)=f\left(x_{2}, Q_{2} ; \ldots ; x_{n}, Q_{n} ; x_{1}+N, Q_{1}\right)
$$

we obtain the additional relation

$$
A_{P_{1}, \ldots, P_{n}}^{Q_{1}, \cdots, Q_{n}}=e^{i k_{P_{1}} N} A_{P_{2}, \ldots, P_{n}, P_{1}}^{Q_{2}, \cdots, Q_{n}, Q_{1}}
$$

which together with (31) should give us the energies.

Successive applications of (31) give us in general distinct relations between the amplitudes. For example $A_{\ldots, k_{1}, k_{2}, k_{3}, \ldots}^{\ldots, \alpha, \beta, \gamma}$ relate to $A_{\ldots, k_{3}, k_{2}, k_{1}, \ldots}^{\ldots, \gamma, \alpha, \ldots}$ by performing the permutations $\alpha \beta \gamma \rightarrow \beta \alpha \gamma \rightarrow \beta \gamma \alpha \rightarrow \gamma \beta \alpha$ or $\alpha \beta \gamma \rightarrow$ $\alpha \gamma \beta \rightarrow \gamma \alpha \beta \rightarrow \gamma \beta \alpha$, and consequently the $S$-matrix should satisfy the Yang-Baxter [19, 29] equation

$$
\begin{gathered}
\sum_{\gamma, \gamma^{\prime}, \gamma^{\prime \prime}=1}^{N} S_{\gamma, \gamma^{\prime}}^{\alpha, \alpha^{\prime}}\left(k_{1}, k_{2}\right) S_{\beta, \gamma^{\prime \prime}}^{\gamma, \alpha^{\prime \prime}}\left(k_{1}, k_{3}\right) \quad S_{\beta^{\prime}, \beta^{\prime \prime}}^{\gamma^{\prime}, \gamma^{\prime \prime}},\left(k_{2}, k_{3}\right)= \\
\\
\sum_{\gamma, \gamma^{\prime}, \gamma^{\prime \prime}=1}^{N} S_{\gamma^{\prime}, \gamma^{\prime \prime}}^{\alpha^{\prime}, \alpha^{\prime \prime}}\left(k_{2}, k_{3}\right) S_{\gamma, \beta^{\prime \prime}}^{\alpha, \gamma^{\prime \prime}}\left(k_{1}, k_{3}\right) S_{\beta, \beta^{\prime}}^{\gamma, \gamma^{\prime}}\left(k_{1}, k_{2}\right),
\end{gathered}
$$

for $\alpha, \alpha^{\prime}, \alpha^{\prime \prime}, \beta, \beta^{\prime}, \beta^{\prime \prime}=1,2, \ldots, N$ and $S$ given by (25). Actually the relation (34) is a necessary and sufficient condition $[19,29]$ to obtain a non-trivial solution for the amplitudes in Eq. (31).

We can verify by a long and straightforward calculation that for arbitrary number of classes $N$ and values of the sizes $s_{1}, s_{2}, \ldots, s_{N}$, the $S$ matrix (25), satisfies the Yang-Baxter equation (34), and consequently we may use relations (31) and (33) to obtain the eigenenergies of the Hamiltonian (6). Applying relation (31) $n$ times on the right of equation (33) we obtain a relation between the amplitudes with the same ordering in the lower indices:

$$
\begin{array}{r}
A_{P_{1}, \ldots, P_{n}}^{Q_{1}, \ldots, Q_{n}}=e^{i k_{P_{1}} N} A_{P_{2}, \ldots, P_{n}, P_{1}}^{Q_{2}, \ldots, Q_{n}, Q_{1}}=\left(\prod_{i=2}^{n}-\Xi_{P_{i}, P_{1}}\right) e^{i k_{P_{1}} N} \sum_{Q_{1}^{\prime}, \ldots, Q_{n}^{\prime} Q_{1}^{\prime \prime}, \ldots, Q_{n}^{\prime \prime}} \sum_{S_{Q_{1}^{\prime}, Q_{1}^{\prime \prime}}^{Q_{1}, Q_{2}^{\prime \prime}}\left(k_{P_{1}}, k_{P_{1}}\right) S_{Q_{2}^{\prime}, Q_{2}^{\prime \prime}}^{Q_{2}, Q_{3}^{\prime \prime}}\left(k_{P_{2}}, k_{P_{1}}\right) \cdots S_{Q_{n-1}^{\prime}, Q_{n-1}^{\prime \prime}}^{Q_{n-1}, Q_{n}^{\prime \prime}}\left(k_{P_{n-1}}, k_{P_{1}}\right) S_{Q_{n}^{\prime}, Q_{n}^{\prime \prime}}^{Q_{n}, Q_{1}^{\prime \prime}}\left(k_{P_{n}}, k_{P_{1}}\right) A_{P_{1}, \ldots, P_{n}}^{Q_{1}^{\prime}, \ldots, Q_{n}^{\prime},}}
\end{array}
$$

where we have introduced the harmless extra sum 


$$
1=\sum_{Q_{1}^{\prime \prime}, Q_{2}^{\prime \prime}=1}^{N} \delta_{Q_{2}^{\prime \prime}, Q_{1}^{\prime}} \delta_{Q_{1}^{\prime \prime}, Q_{1}}=\sum_{Q_{1}^{\prime \prime}, Q_{2}^{\prime \prime}=1}^{N} S_{Q_{1}^{\prime}, Q_{1}^{\prime \prime}}^{Q_{1}, Q^{\prime \prime}}\left(k_{P_{1}}, k_{P_{1}}\right)
$$

(see [26] for illustrations of the above equations). In order to fix the values of $\left\{k_{j}\right\}$ we should solve (35), i.e., we should find the eigenvalues $\Lambda(k)$ of the matrix

$$
T(k)_{\left\{Q^{\prime}\right\}}^{\{Q\}}=\sum_{Q_{1}^{\prime \prime}, \ldots, Q_{n}^{\prime \prime}=1}^{N}\left(\prod_{l=1}^{n} S_{Q_{l}^{\prime}, Q_{l}^{\prime \prime}}^{Q_{l}, Q^{\prime \prime}}\left(k_{P_{l}}, k\right)\right),
$$

with periodic boundary condition

$$
S_{Q_{n}^{\prime}, Q_{n}^{\prime \prime}}^{Q_{n}, Q_{n+1}^{\prime \prime}}\left(k_{P_{n}}, k\right)=S_{Q_{n}^{\prime}, Q_{n}^{\prime \prime}}^{Q_{n}, Q_{1}^{\prime \prime}}\left(k_{P_{n}}, k\right) .
$$

The Bethe-ansatz equations which fix the set $\left\{k_{l}\right\}$ will be given from (35) by

$$
e^{-i k_{j} N}=(-1)^{n-1}\left(\prod_{l=1}^{n} \Xi_{l, j}\right) \Lambda\left(k_{j}\right), \quad j=1, \ldots, n .
$$

The matrix $T(k)$ has dimension $N^{n} \times N^{n}$ and can be interpreted as the transfer matrix of an inhomogeneous $N(2 N-1)$-vertex model in a two dimensional lattice with periodic boundary conditions in the horizontal direction ( $n$ sites). Due to the special form of the S matrix (25) the eigenvalues of (37) are invariant under a local gauge transformation where for each factor $S\left(k_{P_{l}}, k\right)$ in (37):

$$
S_{Q_{l}^{\prime}, Q_{l}^{\prime \prime}}^{Q_{l}, Q_{1+1}^{\prime \prime}}\left(k_{P_{l}}, k\right) \rightarrow S_{Q_{l}^{\prime}, Q_{l}^{\prime \prime}}^{Q_{l}, Q_{l+1}^{\prime \prime}}\left(k_{P_{l}}, k\right) \frac{\phi_{Q_{l+1}^{\prime \prime}}^{(l)}}{\phi_{Q_{l}^{\prime \prime}}^{(l)}},
$$

where $\phi_{\alpha}^{(l)}(l=1, \ldots, L ; \quad \alpha=1, \ldots, N)$ are arbitrary functions. If we perform the transformation (40) with the special choice

$$
\frac{\phi_{\alpha}^{(l+1)}}{\phi_{\alpha}^{(l)}}=e^{-i k_{P_{l}}\left(s_{\alpha}-1\right)}, \quad(l=2,3, \ldots, N),
$$

the equivalent transfer matrix to be diagonalized is given by

$$
\tilde{T}(k)_{\left\{Q^{\prime}\right\}}^{\{Q\}}=e^{-i k \sum_{i=1}^{n}\left(s_{Q_{i}}-1\right)} T_{0}(k)_{\left\{Q^{\prime}\right\}}^{\{Q\}}
$$

where

$$
T_{0}(k)_{\left\{Q^{\prime}\right\}}^{\{Q\}}=\sum_{Q_{1}^{\prime \prime}, \ldots, Q_{n}^{\prime \prime}=1}^{N}\left(\prod_{l=1}^{n} \tilde{S}_{Q_{l}^{\prime}, Q_{l}^{\prime \prime}}^{Q_{l}, Q_{1+1}^{\prime \prime}}\left(k_{P_{l}}, k\right)\right),
$$

with the twisted boundary condition

$$
\tilde{S}_{Q_{n}^{\prime}, Q_{n}^{\prime \prime}}^{Q_{n}, Q_{n}^{\prime \prime}}\left(k_{P_{n}}, k\right)=\tilde{S}_{Q_{n}^{\prime}, Q_{n}^{\prime \prime}}^{Q_{n}, 1}\left(k_{P_{n}}, k\right) \Phi_{Q_{1}^{\prime \prime}}
$$

with twisted phase

$$
\Phi_{l}=e^{i\left(s_{l}-1\right) \sum_{j=1}^{n} k_{j}}, l=1, \ldots, N .
$$

The matrix $\tilde{S}$ in (43) and (44) is obtained from those in (25) by taking the size of all particles equal to unity. In this way the problem is transformed into the evaluation of the eigenvalues of a regular (all particles with size 1) inhomogeneous transfer matrix $T_{0}$ with $n(2 N-1)$ nonzero vertex and twisted boundary condition.

Diagonalization of $T_{0}(k)$

The simplest way to diagonalize $T_{0}$ is through the introduction of the monodromy matrix $\mathcal{M}(k)$ [25], which is a transfer matrix of the inhomogeneous vertex model under consideration, where the first and last link in the horizontal direction are fixed to the values $\mu_{1}$ and $\mu_{n+1}$ $\left(\mu_{1}, \mu_{n+1}=1,2, \ldots, N\right)$, that is

$$
\begin{aligned}
\mathcal{M}_{\left\{Q^{\prime}\right\}, \mu_{1}}^{\{Q\}, \mu_{n+1}}(k)= & \Phi_{\mu_{1}} \sum_{\substack{\mu_{2}, \ldots, \mu_{n}=1 \\
Q_{1}^{\prime}, \mu_{1}}}^{N} \tilde{S}_{Q_{1}}^{Q_{1}, \mu_{2}}\left(k_{P_{1}}, k\right) \tilde{S}_{Q_{2}^{\prime}, \mu_{2}}^{Q_{2}, \mu_{3}}\left(k_{P_{2}}, k\right) \cdots \\
& \cdots \tilde{S}_{Q_{n-1}^{\prime}, \mu_{n-1}}^{Q_{n-1}, \mu_{n}}\left(k_{P_{n-1}}, k\right) \tilde{S}_{Q_{n}^{\prime}, \mu_{n}}^{Q_{n}, \mu_{n+1}}\left(k_{P_{n}}, k\right) .
\end{aligned}
$$

The monodromy matrix $\mathcal{M}_{\left\{Q^{\prime}\right\}, \mu_{1}}^{\{Q\}, \mu_{n+1}}(k)$ has coordinates $\{Q\},\left\{Q^{\prime}\right\}$ in the vertical space ( $N^{n}$ dimensions) and coordinates $\mu_{1}, \mu_{n+1}$ in the horizontal space ( $N^{2}$ dimensions). This matrix satisfies the following important relations

$$
\begin{aligned}
\sum_{\nu_{1}^{\prime}, \mu_{1}^{\prime}=1}^{N} \tilde{S}_{\nu_{1}, \mu_{1}}^{\nu_{1}^{\prime}, \mu_{1}^{\prime}}\left(k^{\prime}, k\right) \mathcal{M}_{\left\{\alpha_{l}\right\}, \mu_{1}^{\prime}}^{\left\{\gamma_{1}\right\}, \mu_{n+1}}(k) \mathcal{M}_{\left\{\gamma_{l}\right\}, \nu_{1}^{\prime}}^{\left\{\beta_{l}\right\}, \nu_{n+1}}\left(k^{\prime}\right) & \\
\sum_{\nu_{n+1}^{\prime}, \mu_{n+1}^{\prime}=1}^{N} \mathcal{M}_{\left\{\alpha_{l}\right\}, \nu_{1}}^{\left\{\gamma_{l}\right\}, \nu_{n+1}^{\prime}\left(k^{\prime}\right)} & \mathcal{M}_{\left\{\gamma_{l}\right\}, \mu_{1}}^{\left\{\beta_{l}\right\}, \mu_{n+1}^{\prime}(k) \tilde{S}_{\nu_{n+1}^{\prime}, \mu_{n+1}^{\prime}}^{\nu_{n+1}, \mu_{n+1}}\left(k^{\prime}, k\right),},
\end{aligned}
$$


for $\mu_{1}, \nu_{1}, \mu_{n+1}, \nu_{n+1}=1,2, \ldots, N$.This relation follows directly from successive applications of the YangBaxter equations (34) (see [26], for a graphical representation of these equations).

In order to exploit relation (47) let us denote the components of the monodromy matrix in the horizontal space by

$$
\begin{aligned}
& A(k)_{\alpha}^{\beta}=\mathcal{M}_{\left\{\alpha_{l}\right\}, \alpha}^{\left\{\gamma_{l}\right\}, \beta}(k), \quad B(k)^{\alpha}=\mathcal{M}_{\left\{\alpha_{l}\right\}, N}^{\left\{\gamma_{l}\right\}, \alpha}(k), \\
& C(k)^{\alpha}=\mathcal{M}_{\left\{\alpha_{l}\right\}, \alpha}^{\left\{\gamma_{l}\right\}, N}(k), \quad D(k)=\mathcal{M}_{\left\{\alpha_{l}\right\}, N}^{\left\{\gamma_{l}\right\}, N}(k),(48)
\end{aligned}
$$

where $\alpha, \beta=1,2, \ldots, N-1$. Clearly the transfer matrix $T_{0}(k)$ of the inhomogeneous lattice with twisted boundary conditions, we want to diagonalize, is given by

$$
T_{0}(k)=\sum_{\alpha=1}^{N-1} A_{\alpha}^{\alpha}(k)+D(k) .
$$

As a consequence of (47) the matrices $A_{\alpha}^{\alpha}, B^{\alpha}, C^{\alpha}$ and $D$ in (48) obey some algebraic relations. By setting $\left(\nu_{1}, \mu_{1}, \nu_{n+1}, \mu_{n+1}\right)=(N, \alpha, \gamma, \beta)$ in $(47)$ we obtain

$$
A_{\alpha}^{\beta}(k) B^{\gamma}\left(k^{\prime}\right)=-\frac{\tilde{S}_{N, \alpha}^{\alpha, N}\left(k^{\prime}, k\right)}{\tilde{S}_{N, \alpha}^{N, \alpha}\left(k^{\prime}, k\right)} B^{\beta}(k) A_{\alpha}^{\gamma}\left(k^{\prime}\right)+\sum_{\alpha^{\prime}, \beta^{\prime}=1}^{N-1} \frac{\tilde{S}_{\alpha^{\prime}, \beta^{\prime}}^{\gamma, \beta}\left(k^{\prime}, k\right)}{\tilde{S}_{N, \alpha}^{N, \alpha}\left(k^{\prime}, k\right)} B^{\alpha^{\prime}}\left(k^{\prime}\right) A_{\alpha}^{\beta^{\prime}}(k),
$$

with $(\alpha, \beta=1, \ldots, N-1)$. By setting $\left(\nu_{1}, \mu_{1}, \nu_{n+1}, \mu_{n+1}\right)=(N, N, N, \alpha)$ we obtain

$$
D(k) B^{\alpha}\left(k^{\prime}\right)=\frac{\tilde{S}_{N, N}^{N, N}\left(k, k^{\prime}\right)}{\tilde{S}_{N, \alpha}^{N, \alpha}\left(k, k^{\prime}\right)} B^{\alpha}\left(k^{\prime}\right) D(k)-\frac{\tilde{S}_{\alpha, N}^{N, \alpha}\left(k, k^{\prime}\right)}{\tilde{S}_{N, \alpha}^{N, \alpha}\left(k, k^{\prime}\right)} B^{\alpha}(k) D\left(k^{\prime}\right),
$$

where $(\alpha=1, \ldots, N-1)$. The diagonalization of $T_{0}(k)$ in (49) will be done by exploiting the above relations. This procedure is known in the literature as the algebraic Bethe ansatz [25]. The first step in this method follows from the identification of a reference state $|\Omega\rangle$, which should be an eigenstate of $A_{\alpha}^{\alpha}(k)$ and $D(k)$, and hence $T_{0}(k)$, but not of $B^{\alpha}(k)$. In the present case a suitable reference state is $|\Omega\rangle=\mid\left\{\alpha_{l}=N\right\}>_{l=1, \ldots, n}$, which corresponds to a state with N-class particles only. It is simple to calculate

$$
\begin{aligned}
& A_{\alpha}^{\beta}(k)\left|\Omega>=a_{\alpha}^{\beta}(k)\right| \Omega>, \quad D(k)|\Omega>=d(k)| \Omega>, \\
& C^{\alpha}(k)\left|\Omega>=0, \quad B^{\alpha}(k)\right| \Omega>=\sum_{i=1}^{n} b_{i}^{\alpha}(k) \mid \Omega_{\alpha}^{(i)}>,
\end{aligned}
$$

where

$$
\begin{aligned}
& a_{\alpha}^{\beta}(k)=\delta_{\alpha, \beta} \Phi_{\alpha} \prod_{i=1}^{n} \tilde{S}_{N, \alpha}^{N, \alpha}\left(k_{P_{i}}, k\right), \quad d(k)=\Phi_{N} \prod_{i=1}^{n} \tilde{S}_{N, N}^{N, N}\left(k_{P_{i}}, k\right), \\
& b_{i}^{\alpha}(k)=\Phi_{N} \prod_{l=1}^{i-1} \tilde{S}_{N, N}^{N, N}\left(k_{P_{l}}, k\right) \prod_{l=i}^{n} \tilde{S}_{N, \alpha}^{N, \alpha}\left(k_{P_{l}}, k\right),
\end{aligned}
$$

and $\left|\Omega_{\alpha}^{(i)}\right\rangle=\mid\left\{\alpha_{l \neq i}=N\right\}, \alpha_{i}=\alpha>$. The matrices $B^{\alpha}(k)$ act as creation operators in the reference ("vacuum") state, by creating particles of class $\alpha(1,2, \ldots, N)$ in a sea of particles of Nth class $\mid \Omega>$. We then expect that the eigenvectors of $T_{0}(k)$ corresponding to $m_{1}(1,2, \ldots, n)$ particles, belonging to classes distinct from $N$, can be expressed as

$$
\left|k_{l}^{(1)} ; F>=\sum_{\{\beta\}} F_{\beta_{1}, \ldots, \beta_{m_{1}}} B^{\beta_{1}}\left(k_{1}^{(1)}\right) B^{\beta_{2}}\left(k_{2}^{(1)}\right) \cdots B^{\beta_{m_{1}}}\left(k_{m_{1}}^{(1)}\right)\right| \Omega>,
$$

where $\left\{k_{l}^{(1)}, l=1, \ldots, m_{1}\right\}$ and $F_{\beta_{1}, \ldots, \beta_{m_{1}}}$ are variables to be fixed by the eigenvalue equation

$$
T_{0}(k)\left|k_{l}^{(1)}, F>=\Lambda^{(0)}(k)\right| k_{l}^{(1)}, F>.
$$

Using (50) successively, and (52),(53) we obtain

$$
\begin{aligned}
& A_{\alpha}^{\alpha}(k) B^{\beta_{1}}\left(k_{1}^{(1)}\right) B^{\beta_{2}}\left(k_{2}^{(1)}\right) \cdots B^{\beta_{m_{1}}}\left(k_{m_{1}}^{(1)}\right)=\sum_{\left\{\alpha_{1}^{\prime}, \ldots, \alpha_{m_{1}}^{\prime}=1\right\}}^{N} \sum_{\left\{\beta_{1}^{\prime}, \ldots, \beta_{m_{1}}^{\prime}=1\right\}}^{N} \\
& \tilde{S}_{\alpha_{1}^{\prime}, \beta_{1}^{\prime}}^{\beta_{1}, \alpha}\left(k_{1}^{(1)}, k\right) \tilde{S}_{\alpha_{2}^{\prime}, \beta_{2}^{\prime}}^{\beta_{2}, \beta_{1}^{\prime}}\left(k_{2}^{(1)}, k\right) \cdots \tilde{S}_{\alpha_{m_{1}-1}^{\prime}, \beta_{m_{1}-1}^{\prime}}^{\beta_{m_{1}-1},_{1}^{\prime}-2}\left(k_{m_{1}-1}^{(1)}, k\right) \tilde{S}_{\alpha_{m_{1}}^{\prime}, \alpha}^{\beta_{m_{1}}, \beta_{m_{1}-1}^{\prime}\left(k_{m_{1}}^{(1)}, k\right)} \\
& \times \Phi_{\alpha} \frac{\prod_{j=1}^{n} \tilde{S}_{N, \alpha}^{N, \alpha}\left(k_{j}, k\right)}{\prod_{j=1}^{m_{1}} \tilde{S}_{N, \alpha}^{N, \alpha}\left(k_{j}^{(1)}, k\right)} B^{\alpha_{1}^{\prime}}\left(k_{1}^{(1)}\right) B^{\alpha_{2}^{\prime}}\left(k_{2}^{(1)}\right) \cdots B^{\alpha_{m_{1}}^{\prime}}\left(k_{m_{1}}^{(1)}\right) \Omega>\quad+\text { "unwanted terms", }
\end{aligned}
$$


where the "unwanted terms" are those ones which are not expressed in the "Bethe basis" produced by the $B^{\alpha}\left(k_{j}^{(1)}\right)$ operators. Similarly, using (51) successively and (52)-(53) we obtain

$$
\begin{aligned}
& D(k) B^{\beta_{1}}\left(k_{1}^{(1)}\right) \cdots B^{\beta_{m_{1}}}\left(k_{m_{1}}^{(1)}\right) \mid \Omega>=\Phi_{N}\left(\prod_{l=1}^{n} \tilde{S}_{N, N}^{N, N}\left(k_{l}, k\right)\right) \\
& \quad \times\left(\prod_{l=1}^{m_{1}} \frac{\tilde{S}_{N, N}^{N, N}\left(k, k_{l}^{(1)}\right)}{\tilde{S}_{N, \beta_{l}}^{N, \beta_{l}}\left(k, k_{l}^{(1)}\right)}\right) B^{\beta_{1}}\left(k_{1}^{(1)}\right) \cdots B^{\beta_{m_{1}}\left(k_{m_{1}}^{(1)}\right) \mid \Omega>\quad+\quad \text { "unwanted terms". }}
\end{aligned}
$$

The relations (56) and (57) when used in (54)-(55) give us

$$
\begin{aligned}
& T_{0}(k) \quad \mid k_{\alpha}^{(1)}, F>=\frac{\prod_{j=1}^{n} \tilde{S}_{N, 1}^{N, 1}\left(k_{j}, k\right)}{\prod_{j=1}^{m_{1}} \tilde{S}_{N, 1}^{N, 1}\left(k_{j}^{(1)}, k\right)} \sum_{\{\beta\}} \sum_{\left\{\alpha^{\prime}\right\}} T_{1}(k)_{\left\{\alpha^{\prime}\right\}}^{\{\beta\}} F_{\{\beta\}} B^{\alpha_{1}^{\prime}}\left(k_{1}^{(1)}\right) \cdots B^{\alpha_{m_{1}}^{\prime}\left(k_{m_{1}}^{(1)}\right) \mid \Omega>} \\
& +\Phi_{N} \prod_{i=1}^{n} \tilde{S}_{N, N}^{N, N}\left(k_{i}, k\right) \sum_{\{\beta\}} \prod_{l=1}^{m_{1}}\left(\frac{\tilde{S}_{N, N}^{N, N}\left(k, k_{l}^{(1)}\right)}{\tilde{S}_{N, \beta_{l}}^{N, \beta_{l}}\left(k, k_{l}^{(1)}\right)}\right) F_{\{\beta\}} B^{\beta_{1}}\left(k_{1}^{(1)}\right) \cdots B^{\beta_{m_{1}}\left(k_{m_{1}}^{(1)}\right) \mid \Omega>} \\
& \text { +"unwanted terms", }
\end{aligned}
$$

where

$$
T_{1}(k)_{\left\{\alpha^{\prime}\right\}}^{\{\beta\}}=\sum_{\alpha=1}^{N-1} \Phi_{\alpha} \sum_{\beta_{1}^{\prime}, \ldots, \beta_{m_{1}}^{\prime}=1}^{N-1} \tilde{S}_{\alpha_{1}^{\prime}, \beta_{1}^{\prime}}^{\beta_{1}, \alpha}\left(k_{1}^{(1)}, k\right)\left(\prod_{i=1}^{m_{1}-1} \tilde{S}_{\alpha_{i}^{\prime}, \beta_{i+1}^{\prime}}^{\beta_{i}, \beta_{i}^{\prime}}\left(k_{i}^{(1)}, k\right)\right) \tilde{S}_{\alpha_{m_{1}}^{\prime}, \alpha}^{\beta_{m_{1}}, \beta_{m_{1}-1}^{\prime}}\left(k_{m}^{(1)}, k\right)
$$

is a $(N-1)^{m_{1}}$-dimensional transfer matrix of a inhomogeneous vertex model, with inhomogeneties $\left\{k_{m_{1}}^{(1)}, k_{m_{1}-1}^{(1)}\right.$ (notice the reverse order of the inohomogeneties, when compared with (43)) and twisted boundary conditions (boundary phases $\Phi_{\alpha}, \alpha=1, \ldots, N-1$ ).

In order to proceed we need now to diagonalize the new transfer matrix $T_{1}(k)$, that is we must solve

$$
\sum_{\{\beta\}} T_{1}(k)_{\left\{\alpha^{\prime}\right\}}^{\{\beta\}} F_{\{\beta\}}=\Lambda^{(1)}(k) F_{\left\{\alpha^{\prime}\right\}}
$$

and then (58) give us

$$
T_{0}(k)\left|k_{\alpha}^{(1)}, F>=\Lambda^{(0)}(k)\right| k_{\alpha}^{(1)}, F>+ \text { "unwanted terms", }
$$

where, using the fact that $\tilde{S}_{N, N}^{N, N}\left(k_{l}, k\right)=1$,

$$
\Lambda^{(0)}(k)=\frac{\prod_{j=1}^{n} \tilde{S}_{N, \alpha}^{N, \alpha}\left(k_{j}, k\right)}{\prod_{j=1}^{m_{1}} \tilde{S}_{N, 1}^{N, 1}\left(k_{j}^{(1)}, k\right)} \Lambda^{(1)}(k)+\Phi_{N} \prod_{l=1}^{m_{1}} \frac{1}{\tilde{S}_{N, 1}^{N, 1}\left(k, k_{l}^{(1)}\right)} .
$$

In order to prove that $\Lambda^{(0)}$ and $\mid K_{\alpha}^{(1)}, F>$ are the eigenvalues and eigenvectors of $T_{0}(k)$, we should fix $\left\{k_{1}^{(1)}, \ldots, k_{m_{1}}^{(1)}\right\}$ by requiring that the "unwanted terms" in (61) vanish. Although for $N=2$ this calculation is not complicated [26] for arbitrary $N$ it is not simple. Since the expression (62) for the eigenvalues should be valid for arbitrary values of $k$ we can obtain $\Lambda^{(1)}\left(k_{j}^{(1)}\right)$ in an alternative way from the following trick [31]. At $k=k_{j}^{(1)}\left(j=1, \ldots, m_{1}\right)$ the denominators of the factors in $(62) \operatorname{vanish}\left(\tilde{S}_{n, l}^{N, l}\left(k_{j}^{(1)}, k_{j}^{(1)}\right)=0, l \neq N\right)$, and since we should have a finite result, we have the conditions

$$
\Lambda^{(1)}\left(k_{j}^{(1)}\right)=\Phi_{N} \prod_{i=1}^{n} \frac{1}{\tilde{S}_{N, 1}^{N, 1}\left(k_{i}, k_{j}^{(1)}\right)} \prod_{l^{\prime}=1, l^{\prime} \neq j}^{m_{1}} \frac{\tilde{S}_{N, 1}^{N, 1}\left(k_{l^{\prime}}^{(1)}, k_{j}^{(1)}\right)}{\tilde{S}_{N, 1}^{N, 1}\left(k_{j}^{(1)}, k_{l^{\prime}}^{(1)}\right)}, j=1, \ldots, m_{1} .
$$

Notice that our result in (63) does not depend on the particular ordering of the additional variables $k_{j}^{(1)}(j=$ $\left.1, \ldots, m_{1}\right)$. This means that if instead of the ordering chosen in (54), we chose the reverse order, namely,

$$
\left|k_{\alpha}^{(1)}, F>=\sum_{\{\beta\}} F_{\beta_{1}, \ldots, \beta_{m_{1}}} B^{\beta_{m_{1}}}\left(k_{m_{1}}^{(1)}\right) B^{\beta_{m_{1}-1}}\left(k_{m_{1}-1}^{(1)}\right) \cdots B^{1}\left(k_{1}^{(1)}\right)\right| \Omega>,
$$

we would obtain the same results (61)-(63), but now $T_{1}$ is the transfer matrix, with boundary condition specified by the phase $\Phi_{\alpha}$, of a problem with $(N-1)$ species and inhomogeneities $k_{1}^{(1)}, \ldots, k_{m_{1}}^{(1)}$ (notice we have now the same 
order of the inhomogeneties as in (43)). This means that the eigenvalue $\Lambda(k)=\Lambda^{(0)}(k)$ of the transfer matrix of the problem with $N$ classes and inhomogeneities $\left(k_{1}^{(0)}, k_{2}^{(0)}, \ldots, k_{n}^{(0)}\right) \equiv\left(k_{1}, k_{2}, \ldots, k_{n}\right)$ is related to the eigenvalue $\Lambda^{(1)}(k)$ of the problem with $(N-1)$ classes and inhomogeneities $k_{1}^{(1)}, k_{2}^{(1)}, \ldots, k_{m_{1}}^{(1)}$. Iterating these calculations we obtain the generalization of the relation (62) and the condition (63)

$$
\begin{aligned}
\Lambda^{(l)}(k)= & \left(\prod_{l^{\prime}=1}^{m_{l}} \tilde{S}_{N, 1}^{N, 1}\left(k_{l^{\prime}}^{(l)}, k\right)\right)\left(\prod_{l^{\prime}=1}^{m_{l+1}} \frac{1}{\tilde{S}_{N, 1}^{N, 1}\left(k_{l^{\prime}}^{(l+1)}, k\right)}\right) \Lambda^{(l+1)}(k)+ \\
& \Phi_{N-l} \prod_{l^{\prime}=1}^{m_{l+1}} \frac{1}{\tilde{S}_{N, 1}^{N, 1}\left(k, k_{l^{\prime}}^{(l+1)}\right)}, l=0,1, \ldots, N-1, \\
\Lambda^{(l+1)}\left(k_{j}^{(l+1)}\right)= & \Phi_{N-l} \prod_{l^{\prime}=1}^{m_{l}}\left(\frac{1}{\tilde{S}_{N, 1}^{N, 1}\left(k_{l^{\prime}}^{(l)}, k_{j}^{(l+1)}\right)}\right) \prod_{l^{\prime}=1, l^{\prime} \neq j}^{m_{l+1}} \frac{\tilde{S}_{N, 1}^{N, 1}\left(k_{l^{\prime}}^{(l+1)}, k_{j}^{(l+1)}\right)}{\tilde{S}_{N, 1}^{N, 1}\left(k_{j}^{(l+1)}, k_{l^{\prime}}^{(l+1)}\right)},
\end{aligned}
$$

which connects the eigenvalues of the inhomogeneous transfer matrix $T_{l}(k)$ and $T_{l+1}(k)$, with inhomogeneities $\left\{k_{j}^{(l)}\right\}$ and $\left\{k_{j}^{(l+1)}\right\}$, related with the problem with $(N-l)$ and $(N-l-1)$ classes of particles, respectively.

However from (39) and (42)-(43), in order to obtain the Bethe-ansatz equations for our original problem we need the eigenvalues of the transfer matrices evaluated at $k_{j}(j=1, \ldots, n)$, i.e., $\Lambda^{(0)}\left(k_{j}\right)$, which are given by

$$
\Lambda^{(0)}\left(k_{P_{j}}\right)=\Phi_{N} \prod_{l=1}^{m_{1}} \frac{1}{\tilde{S}_{N, 1}^{N, 1}\left(k_{P_{j}}, k_{l}^{(1)}\right)}
$$

since $\prod_{j=1}^{n} \tilde{S}_{n, \alpha}^{N, \alpha}\left(k_{j}, k_{P_{j}}\right)=0$. The conditions that fix the variables $\left(k_{j}^{(1)}, j=1, \ldots, m_{1}\right)$ are given by (63). In the left side of this equation we have $\Lambda^{(1)}\left(k_{j}^{(1)}\right)$, which are the eigenvalues of the transfer matrix $T_{1}$ of the model with $(N-1)$ classes of particles and inhomogeneities $\left\{k_{j}^{(1)}, j=1, \ldots, m_{1}\right\}$, evaluated at the partcular point $k_{j}^{(1)}$. This value can be obtained from (65) which gives a generalization of (67)

$$
\Lambda^{(l)}\left(k_{j}^{(l)}\right)=\Phi_{N-l} \prod_{l^{\prime}=1}^{m_{l+1}} \frac{1}{\tilde{S}_{N, 1}^{N, 1}\left(k_{j}^{(l)}, k_{l^{\prime}}^{(l+1)}\right)} \quad(l=0,1, \ldots, N-1) .
$$

The condition (63) is then replaced by

$$
\Lambda^{(1)}\left(k_{j}^{(1)}\right)=\Phi_{N-1} \prod_{l^{\prime}=1}^{m_{2}} \frac{1}{\tilde{S}_{N, 1}^{N, 1}\left(k_{j}^{(1)}, k_{l^{\prime}}^{(2)}\right)}=\Phi_{N} \prod_{i=1}^{n} \frac{1}{\tilde{S}_{N, 1}^{N, 1}\left(k_{i}^{(1)}, k_{j}^{(1)}\right)} \prod_{l^{\prime}=1, l^{\prime} \neq j}^{m_{1}} \frac{\tilde{S}_{N, 1}^{N, 1}\left(k_{l^{\prime}}^{(1)}, k_{j}^{(1)}\right)}{\tilde{S}_{N, 1}^{N, 1}\left(k_{j}^{(1)}, k_{l^{\prime}}^{(1)}\right)},
$$

where now we need to find the relations that fix $\left\{k_{j}^{(2)}\right\}$. Iterating this process we find the generalization of (69)

$$
\begin{aligned}
\Phi_{N-l} & \prod_{l=1}^{m_{l+1}} \frac{1}{\tilde{S}_{N, 1}^{N, 1}\left(k_{j}^{(l)}, k_{l^{\prime}}^{(l+1)}\right)}=\Phi_{N-(l-1)} \prod_{i=1}^{m_{l-1}} \frac{1}{\tilde{S}_{N, 1}^{N, 1}\left(k_{i}^{(l+1)}, k_{j}^{(l)}\right)} \\
& \times \prod_{l^{\prime}=1, l^{\prime} \neq j}^{m_{l}} \frac{\tilde{S}_{N, 1}^{N, 1}\left(k_{l^{\prime}}^{(l)}, k_{j}^{(l)}\right)}{\tilde{S}_{N, 1}^{N, 1}\left(k_{j}^{(l)}, k_{l^{\prime}}^{(l)}\right)},\left(j=1,2, \ldots, m_{l} ; \quad l=0,1, \ldots, N-2\right) .
\end{aligned}
$$

Equations (67) and (70) give us the eigenvalues of the transfer matrix $T_{0}(k)$ evaluated at the points $\left\{k_{j}\right\}$, i. e. $\Lambda^{(0)}\left(k_{j}\right)$. Inserting the above results in $(42)$ and then in (39) we obtain the Bethe-ansatz equations of our original problem.

The eigenenergies of the Hamiltonian (6) in the sector containing $n_{i}$ particles in class $i(i=1,2, \ldots, N)(n=$ $\left.\sum_{j=1}^{N} n_{j}\right)$ and total momentum $p=\frac{2 \pi}{L}(l=0,1, \ldots, L-1)$ are given by

$$
E=-\sum_{j=1}^{n}\left(\epsilon_{-} e^{i k_{j}^{(0)}}+\epsilon_{+} e^{-i k_{j}^{(0)}}-1\right)
$$


where $\left\{k_{j}^{(0)}=k_{j}, j=1, \ldots, n\right\}$ are obtained from the solutions $\left\{k_{j}^{(l)}, l=0, \ldots, N-1 ; j=1, \ldots, m_{l}\right\}$ of the Bethe ansatz equations

$$
\begin{aligned}
e^{i k_{j}\left(L+n-\sum_{i=1}^{N} n_{i} s_{i}\right)} & =(-1)^{n-1} e^{-i p\left(s_{N}-1\right)} \prod_{j^{\prime}=1\left(j^{\prime} \neq j\right)}^{n} \frac{\epsilon_{+}+\epsilon_{-} e^{i\left(k_{j}^{(0)}+k_{j^{\prime}}^{(0)}\right)}-e^{i k_{j}^{(0)}}}{\epsilon^{i\left(k_{j}^{(0)}+k_{j^{\prime}}^{(0)}\right)}-e^{i k_{j^{\prime}}^{(0)}}} \\
& \times \prod_{l=1}^{m_{1}} \frac{\epsilon_{+}\left(e^{i k_{l}^{(1)}}-e^{i k_{j}^{(0)}}\right)}{\epsilon_{+}+\epsilon_{-} e^{i\left(k_{l}^{(1)}+k_{j}^{(0)}\right.}-e^{i k_{j}^{(0)}}} j=1,2, \ldots, n,
\end{aligned}
$$

and

$$
\begin{aligned}
& \prod_{\beta=1}^{m_{l}} \frac{\epsilon_{+}\left(e^{i k_{\alpha}^{(l+1)}}-e^{i k_{\beta}^{(l)}}\right)}{\epsilon_{+}+\epsilon_{-} e^{i\left(k_{\alpha}^{(l+1)}+k_{\beta}^{(l)}\right)}-e^{i k_{\beta}^{(l)}}}=(-1)^{m_{l+1}} e^{i p\left(s_{N-l}-s_{N-l-1}\right)} \prod_{\delta=1}^{m_{l+2}} \frac{\epsilon_{+}\left(e^{i k_{\delta}^{(l+2)}}-e^{i k_{\alpha}^{(l+1)}}\right)}{\epsilon_{+}+\epsilon_{-} e^{i\left(k_{\delta}^{(l+2)}+k_{\alpha}^{(l+1)}\right)}-e^{i k_{\alpha}^{(l+1)}}} \\
& \times \prod_{\alpha^{\prime}=1\left(\alpha^{\prime} \neq \alpha\right)}^{m_{l+1}} \frac{\epsilon_{+}+\epsilon_{-} e^{i\left(k_{\alpha}^{(l+1)}+k_{\alpha^{\prime}}^{(l+1)}\right)}-e^{i k_{\alpha}^{(l+1)}}}{\epsilon_{+}+\epsilon_{-} e^{i\left(k_{\alpha}^{(l+1)}+k_{\alpha^{\prime}}^{(l+1)}\right)}-e^{i k_{\alpha^{\prime}}^{(l+1)}}} \quad l=0,1, \ldots, N-2 ; \quad \alpha=1, \ldots, m_{l},
\end{aligned}
$$

and $m_{l}=\sum_{j=1}^{N-l} n_{j}, l=0, \ldots, N\left(m_{0}=N, m_{N}=0\right)$. It is interesting to observe that in the particular case where $n_{2}=n_{3}=\ldots=n_{N}=0$ we obtain the Betheansatz equations, recently derived [15] (see also [14]), for the asymmetric diffusion problem with particles of size $s_{1}$. Also the case $s_{1}=s_{2}=\ldots=s_{N}=1$ gives us the corresponding Bethe ansatz equations for the standard problem of $N$ types of particles in hierarchical order. The Bethe-ansatz solution in the particular case of $\mathrm{N}=2$ with a a single particle of class 2 $\left(n_{1}=n-1, n_{2}=1\right)$ was derived recently [32]. The Bethe-ansatz equations for the fully asymmetric problem are obtained by setting in (72)-(73) $\epsilon_{+}=1$ and $\epsilon_{-}=0$.

\section{Conclusions and generaliza- tions}

We obtained through the Bethe ansatz the exact solution of the problem in which particles belonging to $N$ distinct classes with hierarchical order diffuse as well interchange positions with rates depending on their relative hierarchy. We show that the exact solution can also be derived in the general case where the particles have arbitrary sizes.

Some extensions of our results can be made. A first and quite interesting generalization of our model happens when we allow molecules in any class to have size $s=0$. Molecules of size zero do not occupy space on the lattice, having no hard-core exclusion effect. Consequently we may have, at a given lattice point, an arbitrary number of them. The Bethe-ansatz solution presented in the previous section is extended di- rectly in this case (the equations are the same) and the eigenenergies are given by fixing in (71)-(73) the appropriate sizes of the molecules. It is interesting to remark that particles of a given class $c^{\prime}(2,3, \ldots, N)$, with size $s_{c^{\prime}}=0$, contrary to the case $s_{c^{\prime}}>1$, where they "accelerate" the diffusion of particles in classes $c<c^{\prime}$, they now "retard" the diffusive motion of these particles. The quantum Hamiltonian in the cases where the particles have size zero is obviously not given by (6) but can be written in terms of spin $S=\infty$ quantum chains. Another further extension of our model is obtained by considering an arbitrary mixture of molecules, where molecules in the same hierarchy may have distinct sizes. The results presented in [15] correspond to the particular case of this generalization where $N=1$ (simple diffusion). For general $N$ the $S$ matrix we obtain in (25) is also a solution of the Yang-Baxter equation (34), but the diagonalization of the transfer matrix of the associated inhomogeneous vertex model is more complicated. The Bethe-ansatz equations in the case of asymmetric diffusion, with particles of unit size $[10,11]$, or with arbitrary size [15], were used to obtain the finite-size corrections of the mass gap $G_{N}$ of the associated quantum chain. The real part of these finite-size corrections is governed by the dynamical critical exponent $z$, i. e.,

$$
\operatorname{Re}\left(G_{N}\right) \sim N^{-z} .
$$

The calculation of the exponent $z$ for the model presented in this paper, with particles of arbitrary sizes, is presently in progress [30].

\section{Acknowledgements}

This work was supported in part by Conselho Nacional de Desenvolvimento Científico e Tecnológico - 
CNPq - Brazil and by the Russian Foundation of Fundamental Investigation ( Grant 99-02-17646).

\section{References}

[1] A. A. Lushnikov, Zh. Éksp. Teor. Fiz. 91, 1376 (1986) [Sov. Phys. JETP 64, 811 (1986)], Phys. Lett. A 120, 135 (1987).

[2] G. Schütz, J. Stat. Phys. 71, 471 (1993).

[3] F. C. Alcaraz, M. Droz, M. Henkel, and V. Rittenberg, Ann. Phys. (N.Y.) 230, 250 (1994).

[4] F. C. Alcaraz and V. Rittenberg, Phys. Lett. B 324, 377 (1993).

[5] F. C. Alcaraz, Int. J. Mod. Phys. B 8, 3449 (1994).

[6] M. D. Grynberg and R. B. Stinchcombe, Phys. Rev. Lett. 74, 1242 (1995).

[7] K. Krebs, M. P. Pfannmüller, B. Wehefritz, and H Henrichsen, J. Stat. Phys. 78, 1429 (1995).

[8] J. E. Santos, G. M. Schütz, and R. B. Stinchcombe, J. Chem. Phys. 105, 2399 (1996)

[9] M. J. de Oliveira, T. Tomé, and R. Dickman, Phys. Rev. A 46, 6294 (1992).

[10] L. H. Gwa and H. Spohn, Phys. Rev. Lett. 68, 725 (1992), Phys. Rev. A 46, 844 (1992).

[11] D. Kim, Phys. Rev. E 52, 3512 (1995).

[12] D. Kim, J. Phys. A 30, 3817 (1997).

[13] F. C. Alcaraz, S. Dasmahapatra and V. Rittenberg, J. Phys. A. 31, 845 (1998).

[14] T. Sasamoto and M. Wadati, J. Phys. A 31, 6057 (1998).

[15] F. C. Alcaraz and R. Z. Bariev, Phys. Rev. E 60, 79 (1999).

[16] B. Derrida, Physics Reports 301, 65 (1998).

[17] T. M. Liggett, Stochastic Interacting Systems: Contact, Voter and Exclusion Process (Springer Verlag, 1999)
[18] G. M. Schütz, Exactly solvable models for many-body systems far from equilibrium in "Phase Transition and Critical Phenomena". Eds. C. Domb and J.L. Lebowitz Vol 19 (to appear).

[19] C. N. Yang, Phys. Rev. Lett. 19, 1312 (1967).

[20] B. Sutherland, Phys. Rev. B 12, 3795 (1975).

[21] S. V. Pokrovskii and A. M. Tsvelick, Zh. Eksp. Teor. Fiz. 93, 2232 (1987)(Sov. Phys.-JETP 66, 6 (1987))

[22] C. Boldrighini, G. Cosimi, S. Frigio, and G. Nuñes, J. Stat. Phys. 55, 611 (1989).

[23] P. A. Ferrari, C. Kipnis and E. Saada, Ann. Prob. 19, 226 (1991)

[24] P. A. Ferrari, Prob. Theory Relat. Fields 91, 81 (1992).

[25] L. A. Takhtajan and L. D. Faddeev, Russ. Math. Surv. 34,11 (1979); V. E. Korepin, I. G. Izergin and N. M. Bogoliubov, Quantum Inverse Scattering Method, Correlation Functions and Algebraic Bethe Ansatz (Cambridge University Press, Cambridge, 1992).

[26] F. C. Alcaraz and R. Z. Bariev, Braz. J. Phys. 30,13 (2000)

[27] B. Derrida, M. R. Evans, V. Hakim, and V. Pasquier, J. Phys. A 26, 1493 (1993).

[28] K. Mallick, S. Mallick and N. Rajewsky, J. Phys. A 32, $48(1999)$

[29] R. J. Baxter, Exactly Solved Models in Statistical Mechanics (Academic Press, New York, 1982).

[30] F. C. Alcaraz and R. Z. Bariev, (to be published).

[31] P. P. Kulish and N. Yu. Reshetikhin, Zh. Eksp. Teor. Fiz. 80, 214 (1981); C. L. Schultz, Physica A 122, 71 (1983); M. Gaudin La fonction d'onde Bethe (Paris, Masson, 1983).

[32] B. Derrida and M. R. Evans, J. Phys. A 32, 4833 (1999). 